\title{
ANALYSIS OF CRUSHING RESPONSE OF COMPOSITE CRASHWORTHY STRUCTURES
}

\author{
M. David and A.F. Johnson \\ *German Aerospace Center (DLR), Institute of Structures and Design \\ Pffaffenwaldring, 38-40, Stuttgart, Germany \\ e-mail: matthew.david@dlr.de, alastair.johnson@dlr.de
}

Key words: composite structures, crashworthiness, energy absorption, crush test method, composites failure modes

\begin{abstract}
The increase usage of polymer composite materials in the aerospace and automotive industry has generated considerable interest in using composite materials for crashworthy structures that absorb impact energy through a controlled failure in progressive crushing. By tailoring the fiber type, matrix type, fiber-matrix interface, fiber stacking sequence and fiber orientation, composite crashworthy structures have been shown to have excellent energy absorption performance characteristics. To understand the energy absorption and failure mechanisms of crashworthy structures, DLR has developed a chamfered tube segment specimen, which is easy to fabricate and gives reproducible axial crush failures under quasistatic and dynamic loading conditions. This may be used for screening of different energy absorbing composite materials and provides design data for crashworthy design studies, see [1].
\end{abstract}

The paper describes a successful methodology for crush testing of chamfered tube segment specimens in both quasi-static and dynamic loading conditions with an analysis method to compare crushing characteristics and energy absorption performance. The specimen is a flanged circular segment which is self-stabilising without edge supports and when triggered fails by steady crushing without buckling, as seen in Fig. 1. The specimens are easily fabricated in open moulds and are clamped in the test at their base, which avoids special support fixtures used in similar tests with flat plate specimens. A test programme is described [2] which compares quasi-static crush behaviour with tests carried out at impact velocities of $2-10 \mathrm{~m} / \mathrm{s}$ in a high rate servo-hydraulic test machine. Tests on carbon fibre fabric/epoxy test specimens are presented in detail, with particular emphasis on the influence of loading rate on energy absorbed and crush failure mechanisms. The testing methodology included the use of a high speed camera to capture the crushing behaviour of the specimen during the crush tests. In addition to capturing the crushing behaviour of the specimen during the crush tests, Highresolution Computed Tomography (HRCT) scanning of the specimen was performed to enable a detailed analysis of crushed specimen using the nanotom ${ }^{\circledR}$ CT system by phoneix|xray. This permits detailed observation of the composites failure surface by X-ray without the need for sectioning and microscopic analysis. The quasi-static crush specimen exhibited the most favourable energy absorption performances in terms of Crush force 
efficiency (CE), Energy Absorbed (EA) and Specific Energy Absorbed (SEA) when compared to the test specimens in the dynamic crush tests. Dynamic EA was typically $20 \%$ below quasi-static values, which is a significant reduction in the design of composite crash absorber structures. To further understand the influence of loading rate on energy absorbed and crush failure mechanisms, a thermal investigation of the crushing process was performed. This Passive Infrared (PIR) imaging technique was successfully implemented in the investigation to quantitatively study the energy absorption capabilities.

Differences in the crushing process and therefore the energy absorption performances between the quasi-static and dynamic crushing process were investigated by CT tests on crushed specimens. Differences were found in the behavior of the splayed lamina bundles during the two crushing process. In the quasi-static crushing process, the splayed lamina bundles exhibit axial splitting and petalling with frond formation due to equally spaced axial cracks along the circumference of the composite segment, as seen in Fig. 2. Frond formation is initiated by a central delamination crack at the crush front, Fig. 2. In the dynamic crushing process however, the splayed lamina bundles fracture at their base with no axial splitting and therefore greater fragmentation of the test specimen was observed at the crush front, with reduced delamination. The different behavior of the splayed lamina bundles can be attributed to the specimen responding in a global mode during the quasi-static crush test and in a local mode during the dynamic crush tests. Coupled with this different crush response mode and the matrix properties which are strain rate sensitive the crushing behaviour of the test specimens varied from the quasi-static crush test and the dynamic crush test.
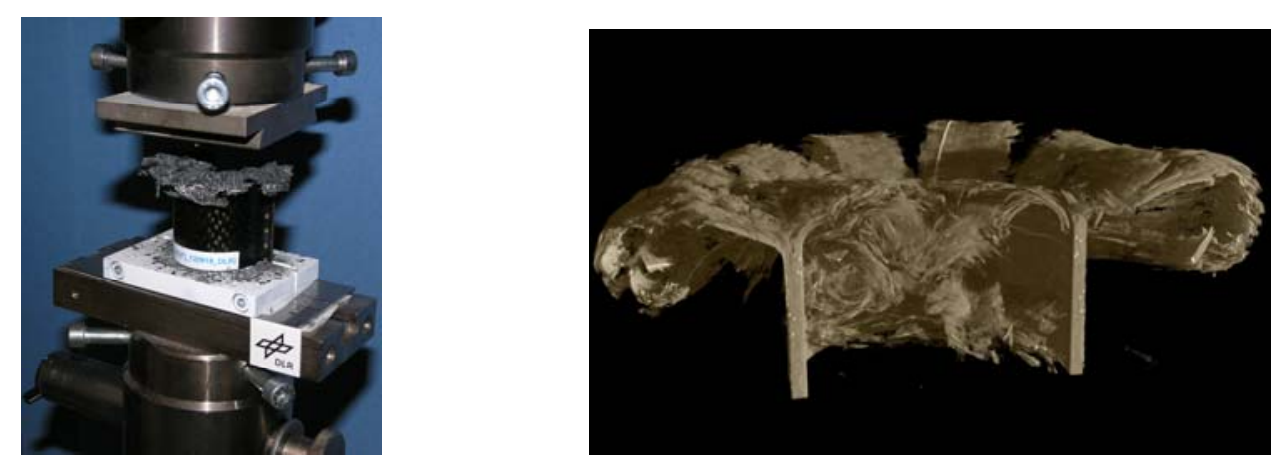

Fig. 1: Composite segment crush test Fig. 2: CT scan of crushed segment specimen

\section{REFERENCES}

[1] Johnson, A.F., Kohlgrüber, D., Design and Performance of Energy Absorbing Subfloor Structures in Aerospace Applications, I. Mech. E. Seminar, London, 2000.

[2] David, M.., Dynamic Crush Tests on Composite Segment Specimens, DLR Report IB 435-2009/11, 2009. 